$\Rightarrow$ BREAST CANCER

\title{
Obesity - tipping the scales of resistance
}

\section{6}

The findings offer insight into the limited efficacy of anti-angiogenic therapy
Phase III trials have not shown a survival benefit for anti-vascular endothelial growth factor (VEGF) therapy in breast cancer, making the pursuit of resistance mechanisms imperative. Although the effect of obesity on the response to anti-angiogenics in breast cancer is unknown, during obesity, hypoxic adipose tissues can release angiogenic and pro-inflammatory factors implicated in resistance. Now, Incio et al. show that obesity promotes resistance to anti-VEGF therapy in breast cancer via upregulation of two such factors - interleukin-6 (IL-6) and fibroblast growth factor 2 (FGF2).

The investigators first assessed the effect of obesity on the response to anti-VEGF antibody bevacizumab in 99 patients with breast cancer enrolled in a neoadjuvant phase II trial that reported a limited response. Interestingly, patients with a high body mass index (BMI) presented with $\sim 33 \%$ larger tumours than those with a normal BMI, and tumours from patients with excess adiposity at baseline were the most hypoxic and the least sensitive to bevacizumab. Importantly, in patients with high adiposity, levels of IL-6 and FGF2 were markedly elevated in plasma (at baseline and throughout therapy) and adipocyte-rich intratumoural regions.

Next, the role of diet-induced obesity in response to anti-VEGF therapy was evaluated in mouse models of breast cancer. Although the growth of established orthotopic tumours was identical in control-treated lean and obese mice, anti-VEGF antibody (B20) treatment was more effective at abrogating tumour growth in lean mice than in obese mice, suggesting that obesity reduces sensitivity to B20. Mirroring the clinical findings, tumours from obese mice were more hypovascularized and hypoxic and harboured a higher abundance of hypertrophic cancer-associated adipocytes (CAAs) than tumours from lean mice. Notably, tumours from B20-treated obese mice had extensive necrosis in CAA-poor regions and limited necrotic tissue in CAA-rich regions, suggesting a protective effect of CAAs on the anti-angiogenic response.

Consistent with clinical observations, tumours from obese mice had markedly higher expression of IL-6 - produced by CAAs and infiltrating myeloid cells - than those from lean mice, particularly in CAA-rich, hypoxic regions. Accordingly, increased activation of signal transducer and activator of transcription 3 (STAT3) was detected in tumour cells, suggesting that IL-6 downstream signalling might mediate obesity-induced resistance to anti-VEGF therapy. Indeed, the combination of an IL-6 inhibitor with B20 reduced tumour growth and metastasis in obese mice to levels comparable to those of lean mice, decreased tumour proliferation and

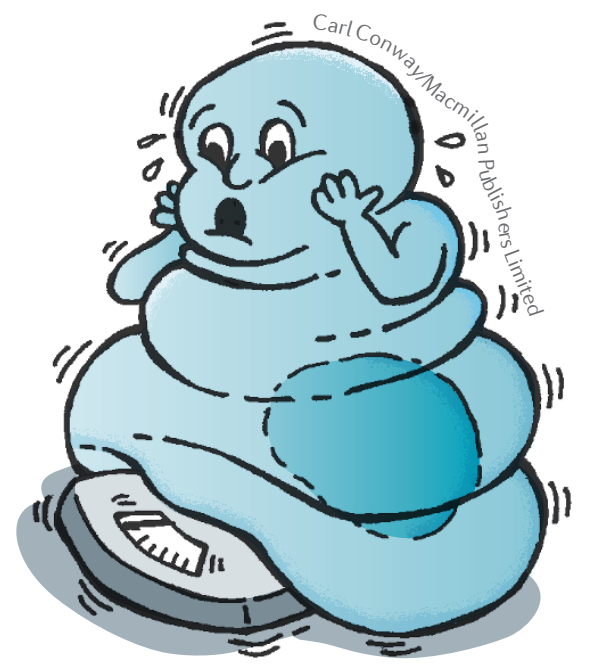

hypoxia, increased perfused vessel density and attenuated immunosuppressive $\mathrm{T}$ cell infiltration.

In a second mouse model, increased expression of FGF2, but not IL-6, was observed in tumours from obese mice - particularly in areas rich in CAAs and cancerassociated fibroblasts - compared with lean mice. Similar to the first model, treatment with an FGF receptor inhibitor or metformin - an anti-diabetes drug that reduces FGF2 expression - improved B20 response in obese, but not lean, tumourbearing mice, suggesting that FGF2 might also mediate obesity-induced resistance to anti-VEGF therapy in the absence of IL- 6 upregulation.

Overall, the study reveals that obesity mediates resistance to antiVEGF therapy in breast cancer via upregulation of IL-6 and, potentially, FGF2 in hypoxic adipocyte-rich intratumoural regions. The findings offer insight into the limited efficacy of anti-angiogenic therapy in patients with breast cancer and suggest that combinatorial therapeutic strategies could overcome resistance in the clinical setting.

Conor A. Bradley

ORIGINAL ARTICLE Incio, J. et al. Obesity promotes resistance to anti-VEGF therapy in breast cancer by up-regulating IL-6 and potentially FGF- 2 . Sci. Transl Med. 10, eaag0945 https://doi.org/ 10.1126/scitranslmed.aag0945 (2018) 